\title{
Strange quark matter and quark stars with the Dyson-Schwinger quark model
}

\author{
H. Chen ${ }^{1}$, J.-B. Wei ${ }^{1}$, and H.-J. Schulze ${ }^{2}$ \\ 1 School of Mathematics and Physics, China University of Geosciences, Lumo Road 388, 430074 Wuhan, China \\ 2 INFN Sezione di Catania, Dipartimento di Fisica, Universitá di Catania, Via Santa Sofia 64, 95123 Catania, Italy
}

Received: date / Revised version: date

\begin{abstract}
We calculate the equation of state of strange quark matter and the interior structure of strange quark stars in a Dyson-Schwinger quark model within rainbow or Ball-Chiu vertex approximation. We emphasize constraints on the parameter space of the model due to stability conditions of ordinary nuclear matter. Respecting these constraints, we find that the maximum mass of strange quark stars is about 1.9 solar masses, and typical radii are 9-11 km. We obtain an energy release as large as $3.6 \times 10^{53} \mathrm{erg}$ from conversion of neutron stars into strange quark stars.
\end{abstract}

PACS. XX.XX.XX No PACS code given

\section{Introduction}

The hypothesis of stable strange quark matter (SQM) 1 [2,3] and strange quark stars (SQSs) [4,5,6, has been attracting interest since some time. Originally it was demonstrated that in a wide region of the MIT bag model parameter space, SQM, but not nuclear matter, could form the ground state of baryonic matter. Since then the SQM hypothesis has been addressed in numerous articles, see [7,8, 9] for an overview.

The most probable way to observe SQM is in compact stars. Compact stars built entirely of quark matter (QM) were studied in different levels of sophistication, starting with an equation of state (EOS) of a free degenerate Fermi gas of $u, d, s$ quarks with equal masses. Then the structure of SQSs was examined in detail in MIT-bag-type models, taking into account the strange quark mass, the lowestorder correction from the QCD interaction, etc. 10,11. More work on the EOS of SQM, the formation of SQSs, their neutrino emission, rotation, superfluidity, pulsations, electromagnetic radiation, and cooling were also done, see the reviews 7, 8, 12,13. QM in the interior of massive neutron stars (NSs) is one of the current main issues in the physics of compact stars, due to the recent observations of two NSs of about two solar masses, PSR J1614$2230\left(M / M_{\odot}=1.93 \pm 0.02\right)[14,15$ and PSR J0348+0432 $\left(M / M_{\odot}=2.01 \pm 0.04\right)[16$.

The EOS of QM is crucial for the study of SQM and SQSs. Many works have been done to go beyond the MIT bag model, e.g., using perturbative QCD [17, 18, 19, 20, 21, the density-dependent-quark-mass model [22, 23, 24, 25, 26], the Nambu-Jona-Lasino model [27, 28, 29, 30, 31, 32, the chiral quark meson model 33 , or the quasi-particle model [34,35, 36]. However, the EOS remains poorly known due to the nonperturbative character of QCD. The DysonSchwinger equations (DSE) provide a continuum approach to QCD that can simultaneously address both confinement and dynamical chiral symmetry breaking [37,38. They have been applied with success to hadron physics in vacuum 39, 40, 41, 42, 43, 44, 45, and to QCD at nonzero temperature and chemical potential [46, 47, 48, 49, 50, 51, $52,53,54,55,56,57,58,59,60,61,62,63,64,65,66$. Both MIT and NJL model have been recognized as limiting cases of the DSM [31,32].

In this paper, we use a Dyson-Schwinger model (DSM) for QM based on our previous work 62,63,64,65, in which the hadron-quark phase transition in compact stars and the structure of hybrid stars were investigated, in combination with a nuclear matter EOS within the BruecknerHartree-Fock (BHF) many-body approach [67,68, 69, 70]. However, there are still free parameters due to uncertainties of the effective interaction and vacuum pressure in our model, and we will scan its parameter space to investigate the possibility of SQM and SQSs.

The paper is organized as follows. In section 2 we briefly discuss the DSM for QM and the parameters in our model. In section 3.1 we analyze the allowed parameter space for stable SQM, and the corresponding EOS. In section 3.2 we present results on the structure of SQSs, as well as the energy release from conversion of NSs into SQSs. Section 4 contains our conclusions. 


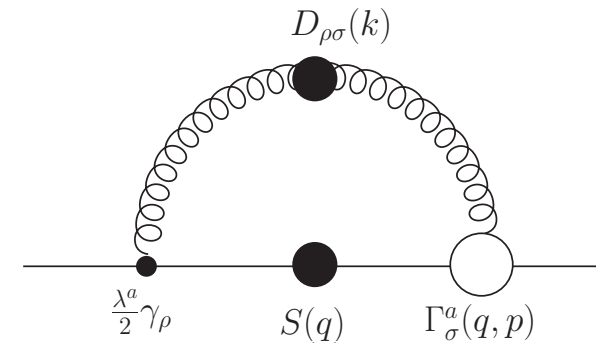

Fig. 1. (Color online) Diagrammatic representation of the quark self-energy, Eq. (1), in the DSM.

\section{Formalism: Quark matter with the Dyson-Schwinger model}

For cold dense QM, we adopt a model based on the DSE of the quark propagator, described in detail in our previous papers $62,63,64,65$. In the following, we only give a brief introduction to the model. We start from the gap equation for the quark propagator $S(p ; \mu)$ at finite chemical potential $\mu \equiv \mu_{q}=\mu_{B} / 3$, depicted in Fig. 1.

$$
\Sigma(p ; \mu)=\int \frac{\mathrm{d}^{4} p}{(2 \pi)^{4}} S(q ; \mu) \frac{\lambda^{a}}{2} \gamma_{\rho} D_{\rho \sigma}(k ; \mu) \Gamma_{\sigma}^{a}(q, p ; \mu),
$$

where $\lambda^{a}$ are the Gell-Mann matrices, $k=p-q, D_{\rho \sigma}(k ; \mu)$ is the dressed gluon propagator, and $\Gamma_{\sigma}^{a}(q, p ; \mu)$ the dressed quark-gluon vertex at finite chemical potential. To solve the equation, one requires an Ansatz for both $D_{\rho \sigma}$ and $\Gamma_{\sigma}^{a}$.

In our model, the combined Ansatz for $D_{\rho \sigma}$ and $\Gamma_{\sigma}^{a}$ is parameterized as

$$
D_{\rho \sigma}(k) \Gamma_{\sigma}^{a}(q, p)=\mathcal{G}(k) D_{\rho \sigma}^{\mathrm{free}}(k) \frac{\lambda^{a}}{2} \Gamma_{\sigma}(q, p)
$$

wherein $D_{\rho \sigma}^{\text {free }}(k)=\left(\delta_{\rho \sigma}-\frac{k_{\rho} k_{\sigma}}{k^{2}}\right) \frac{1}{k^{2}}$ is the Landau-gauge free gluon propagator and $\Gamma_{\sigma}(q, p)$ represents the tensor structure of the quark-gluon vertex Ansatz, while other dressing effects of both the vertex and the gluon propagator are assumed to depend only on the gluon momentum $k$ and are included in a model effective interaction $\mathcal{G}(k)$. Herein we neglect many effects, such as the violation of Lorentz covariance of the gluon propagator at finite chemical potential, the possibility of color-superconductivity [57, 58, 59, etc.

For $\Gamma_{\sigma}$, three forms were investigated in our previous work 64, 65: (1) the bare vertex, also called rainbow (RB) approximation. (2) the Ball-Chiu (BC) vertex, which satisfies the Ward-Takahashi identity of QED and is free of kinetic singularity. The form of the $\mathrm{BC}$ vertex in vacuum was given in 71, and was extended to finite chemical potential in 61. (3) the 1BC vertex, which includes only part of the BC vertex, but is numerically quite similar to the RB approximation. Therefore, in this work we will only present results with the RB and $\mathrm{BC}$ approximations.

For the effective interaction, we employ an infrareddominant interaction modified by the quark chemical po-

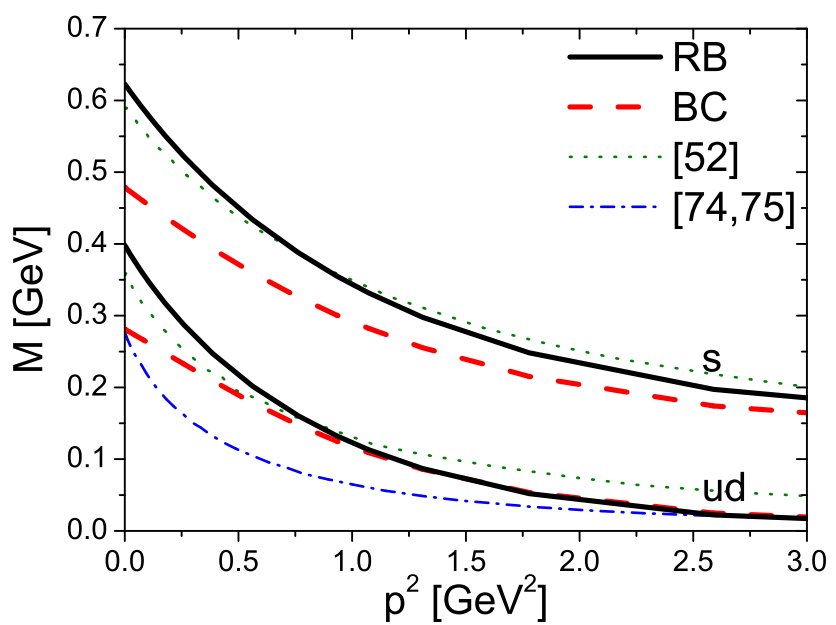

Fig. 2. (Color online) Quark propagator vacuum mass function $M\left(p^{2}\right)$, Eq. (4), for $u, d$ and $s$ quarks and for different vertex Ansätze $\mathrm{RB}$ and $\mathrm{BC}$, in comparison with results of Refs. 52] and 74,75].

tential $62,63,72$

$$
\frac{\mathcal{G}(k)}{k^{2}}=4 \pi^{2} d \frac{k^{2}}{\omega^{6}} e^{-\frac{k^{2}+\alpha \mu^{2}}{\omega^{2}}} .
$$

The parameters $\omega$ and $d$ can be obtained by fitting meson properties and chiral condensate in vacuum [41,42, 73. and we use $\omega=0.5 \mathrm{GeV}, d=1 \mathrm{GeV}^{2}$ (with RB), $d=0.5 \mathrm{GeV}^{2}$ (with $\mathrm{BC}$ ). We choose the quark masses $m_{u, d}=0$ and $m_{s}=115 \mathrm{MeV}$. (We discuss later a possible variation of $m_{s}$ ). The phenomenological parameter $\alpha$ is of particular importance in our work, since it represents a reduction rate of the effective interaction with increasing chemical potential. However, it cannot yet be fixed independently. Obviously, $\alpha=\infty$ corresponds to a noninteracting system at finite chemical potential, i.e., a simple version of the MIT bag model, but in previous and present work we investigate the full parameter space $0<\alpha<\infty$. With varying value of $\alpha$, we can investigate the role of the interaction strength and confront it with different mechanism from other phenomenological models. For example, comparing with the quasi-particle model, we found that the effects of the interaction on light quarks are not in the form of an effective mass, but a modification of the vector part of the quark propagator 62,63 .

The general structure of the quark propagator at finite chemical potential is

$S(p ; \mu)^{-1}=i \boldsymbol{\gamma} \boldsymbol{p} A\left(p^{2}, p_{4}\right)+B\left(p^{2}, p_{4}\right)+i \gamma_{4}\left(p_{4}+i \mu\right) C\left(p^{2}, p_{4}\right)$,

where $A, B, C$ are complex scalar functions and $C=A$ at $\mu=0$. The mass function $M \equiv B / A$ is of particular interest for physical interpretation (asymptotic freedom and dynamical mass generation), and we show the quantity $M\left(p^{2}\right)$ of the Nambu (confined) solution at $\mu=0$ in Fig. 2 in comparison with the corresponding results of Refs. [52,74, 75], which are qualitatively similar. The 


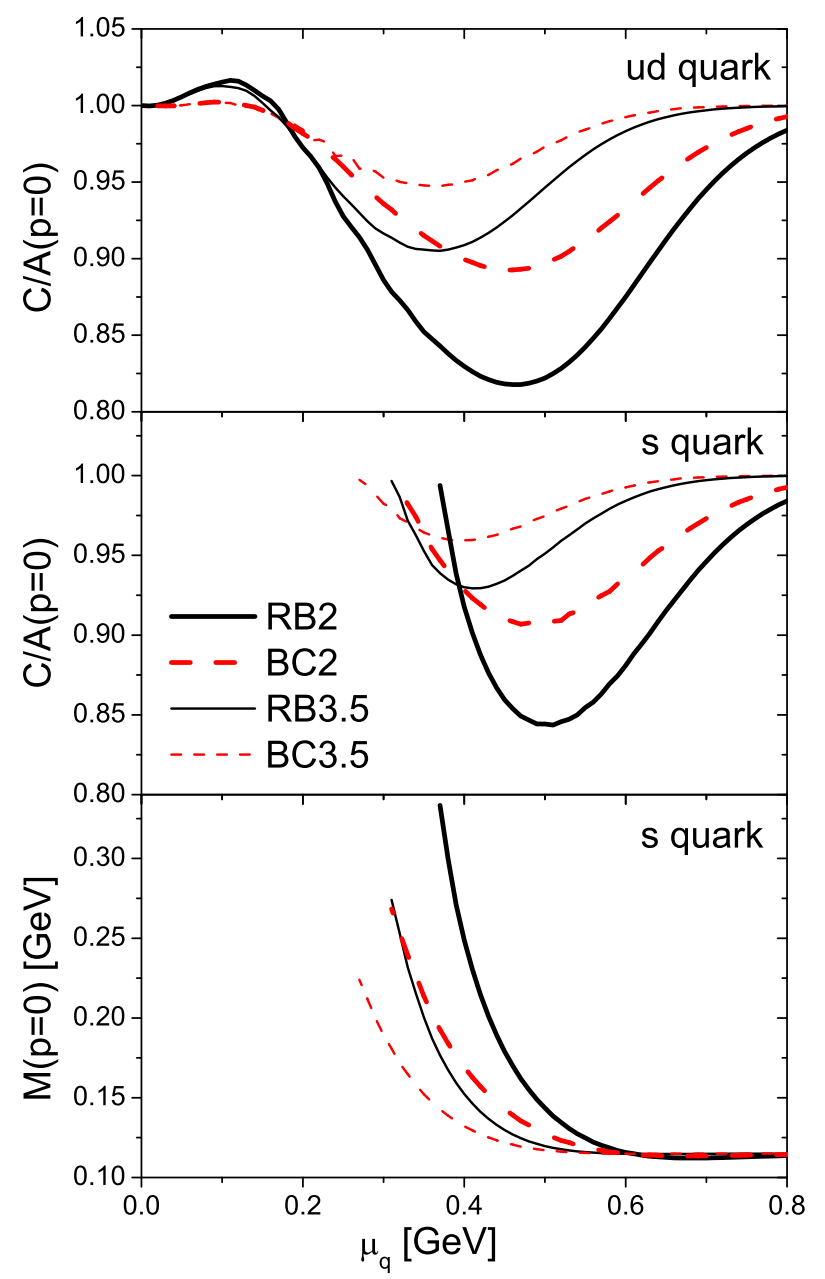

Fig. 3. (Color online) Wigner solutions of the quark propagator components $C / A$ and $M$ at $p=0$ for $u, d$ and $s$ quarks at finite chemical potential for different vertex Ansätze RB and $\mathrm{BC}$ and for $\alpha=2,3.5$, using the notation $\mathrm{RB} \alpha, \mathrm{BC} \alpha$.

latter reference also contains a confrontation with lattice QCD results. Results with BC vertex feature reduced interaction effects and lower mass functions.

At finite chemical potential the discussion of the propagator becomes more complex, due to the additional dependence on $p_{4}$ and the presence of the function $C$. In this work we just illustrate the behavior in Fig. 3 by showing the dependence on chemical potential of the components $C / A$ and $M=B / A$ at $p=0$ for the Wigner (deconfined) solution. As is shown in [76,77, the Wigner solution exists always for massless quarks (upper panel), but for massive strange quarks (lower panels) only at high enough chemical potential, as is seen in the figure. At low chemical potential only the Nambu solution exists, which is characterized by $C \equiv A$ and quite large effective mass 61. For massless $u, d$ quarks one has $B=M=0$, and one can see that $C / A(p=0)<1$, apart from a small region at low chemical potential. This causes the effective Fermi momentum, and consequently the quark number density, to be lower than for free quarks at the same chemical po- tential 61,62,63. For the strange quarks, both $C / A<1$ and $M>m_{s}$ lead to the same effects on the quark number density. Choosing the BC Ansatz or increasing the parameter $\alpha$ leads to weaker interaction effects, as can be seen clearly.

All the relevant thermodynamical quantities of cold QM can be computed from the quark propagator at finite chemical potential, except a boundary value of the pressure $P$, which is represented by a phenomenological bag constant $B_{\mathrm{DS}}$ that we consider another model parameter,

$$
P\left(\mu_{u}, \mu_{d}, \mu_{s}\right)=-B_{\mathrm{DS}}+\sum_{q=u, d, s} \int_{\mu_{q}^{0}}^{\mu_{q}} \mathrm{~d} \mu n_{q}(\mu),
$$

where the density distributions $n_{q}$ are obtained from the quark propagator $61,62,63,78$,

$$
\begin{aligned}
n_{q}(\mu) & =6 \int \frac{d^{3} p}{(2 \pi)^{3}} f_{q}(|\boldsymbol{p}| ; \mu), \\
f_{q}(|\boldsymbol{p}| ; \mu) & =\frac{1}{4 \pi} \int_{-\infty}^{\infty} d p_{4} \operatorname{tr}_{\mathrm{D}}\left[-\gamma_{4} S_{q}(p ; \mu)\right],
\end{aligned}
$$

and the trace is over spinor indices only. Detailed results for the density distributions were shown and discussed in Refs. 62, 63, 64, 65.

In principle, $B_{\mathrm{DS}}$ can be obtained from the pressure difference between the deconfined (Wigner) phase and the confined (Nambu) phase in vacuum, i.e.,

$$
B_{\mathrm{DS}}=\sum_{q=u, d, s}\left[P_{q}^{(c)}\left(\mu_{q}=0\right)-P_{q}^{(d)}\left(\mu_{q}^{0}\right)\right] .
$$

In the framework of DSEs, one usually uses the 'steepestdescent' approximation [79] to calculate the pressure

$$
P[S]=\operatorname{Tr} \operatorname{Ln}\left[S^{-1}\right]-\frac{1}{2} \operatorname{Tr}[\Sigma S],
$$

and in this way we obtain at $\mu=0$ a pressure difference for massless quarks within RB approximation 61]

$$
P_{u, d}^{(c)}(\mu=0)-P_{u, d}^{(d)}(\mu=0) \approx 45 \mathrm{MeV} \mathrm{fm}^{-3} .
$$

Interpreting the Nambu phase as the real vacuum with $P_{u, d}^{(c)}(\mu=0)=0$, we then obtain the pressure of the Wigner phase for light quarks in vacuum $P_{u, d}^{(d)}\left(\mu_{0}=0\right) \approx$ $-45 \mathrm{MeV} \mathrm{fm}^{-3}$ and the effective bag constant from contributions of $u$ and $d$ quarks as $B_{\mathrm{DS}}^{n_{f}=2} \approx 90 \mathrm{MeV} \mathrm{fm}^{-3}$. This value was used in our previous work. However, such an approximation is only consistent with the RB approximation and a static gluon propagator. There is no Wigner solution for strange quarks at $\mu=0$ [76, 80. With the introduction of the parameter $\alpha$ only in the Wigner phase, the steepest-descent approximation is not consistent with the gap equation at finite chemical potential. So we cannot obtain $P_{s}\left(\mu_{s, 0}\right)$ and its contribution to $B_{\mathrm{DS}}$ is unclear. Furthermore, the steepest-descent approximation is not consistent with the $\mathrm{BC}$ vertex either. Therefore, in this paper we allow a free variation of $B_{\mathrm{DS}}$, but expecting it to be of the same order as $90 \mathrm{MeV} \mathrm{fm}^{-3}$. In the following, $B_{\mathrm{DS}}$ is always given in units of $\mathrm{MeV} \mathrm{fm}^{-3}$ in the text and figures. 


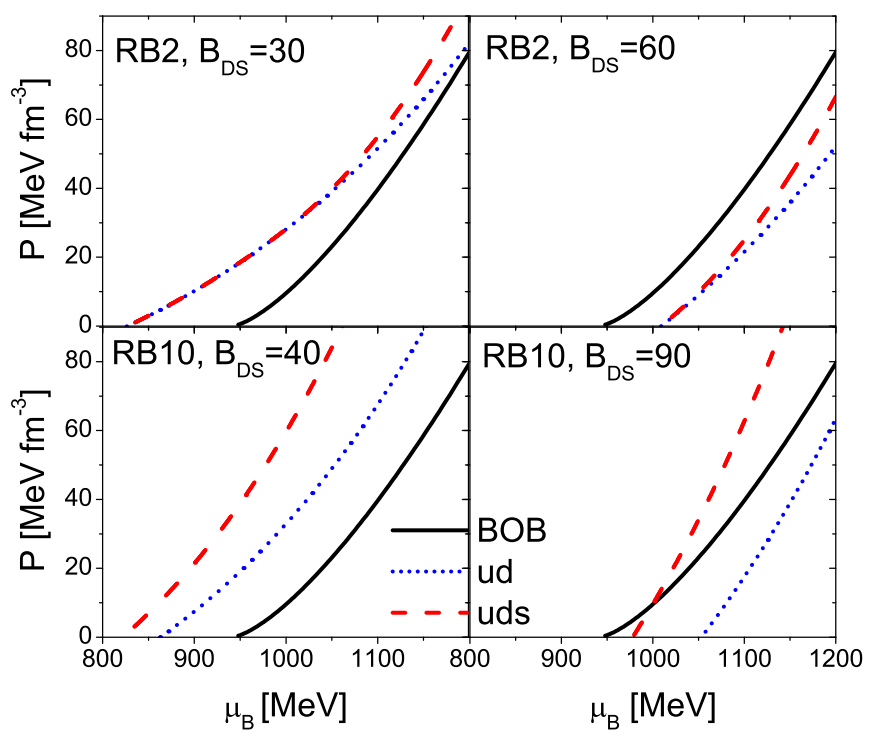

Fig. 4. (Color online) Pressure vs. baryon chemical potential of dense NS matter for different models [nuclear matter (BOB), 2-flavor (ud) and 3-flavor (uds) quark matter] and parameters [notation $\mathrm{RB} \alpha$ indicates $\mathrm{RB}$ approximation with density parameter $\alpha ; B_{\mathrm{DS}}$ the value of the bag constant in $\mathrm{MeV} \mathrm{fm}^{-3}$ ].

\section{Results and discussion}

\subsection{Stability of strange quark matter}

Since SQM would probably appear in the form of SQSs 2], we investigate in the following NS matter, i.e., cold, neutrino-free, charge-neutral, and beta-stable SQM 62, 63, characterized by two degrees of freedom, the baryon and charge chemical potentials $\mu_{B}$ and $\mu_{Q}$. The corresponding equations are

$$
\mu_{i}=b_{i} \mu_{B}+q_{i} \mu_{Q}, \quad \sum_{i} q_{i} \rho_{i}=0,
$$

$b_{i}$ and $q_{i}$ denoting baryon number and charge of the particle species $i=n, p, e, \mu$ in the nuclear phase and $i=$ $u, d, s, e, \mu$ in the quark phase, respectively.

In Fig. 4 we first illustrate the corresponding EOS $P\left(\mu_{B}\right)$ in our DSM with the RB approximation ('uds', dashed red curves) with various typical values of the parameters $\alpha$ and $B_{\mathrm{DS}}$ (different panels $\mathrm{RB} \alpha-B_{\mathrm{DS}}$ ), in comparison with the EOS of two-flavor QM (2QM) ('ud', dotted blue curves), as well as that of hadronic nuclear matter from the Brueckner-Bethe-Goldstone theory with the Bonn-B potential 70] ('BOB', solid black curve). At given chemical potential $\mu_{B}$, the physically realized phase is the one with the highest pressure $P$.

With a parameter $\alpha=2$ (upper panels), i.e., a moderate reduction rate of the interaction strength, strange quarks appear only at very large chemical potential due to their large dynamical mass. In such cases, stable matter with zero pressure can only be $2 \mathrm{QM}$ (upper left panel) for a small effective bag constant $B_{\mathrm{DS}}$, or nuclear matter (upper right panel) for a large $B_{\mathrm{DS}}$. An increasing value of $B_{\mathrm{DS}}$ reduces the stability of QM.

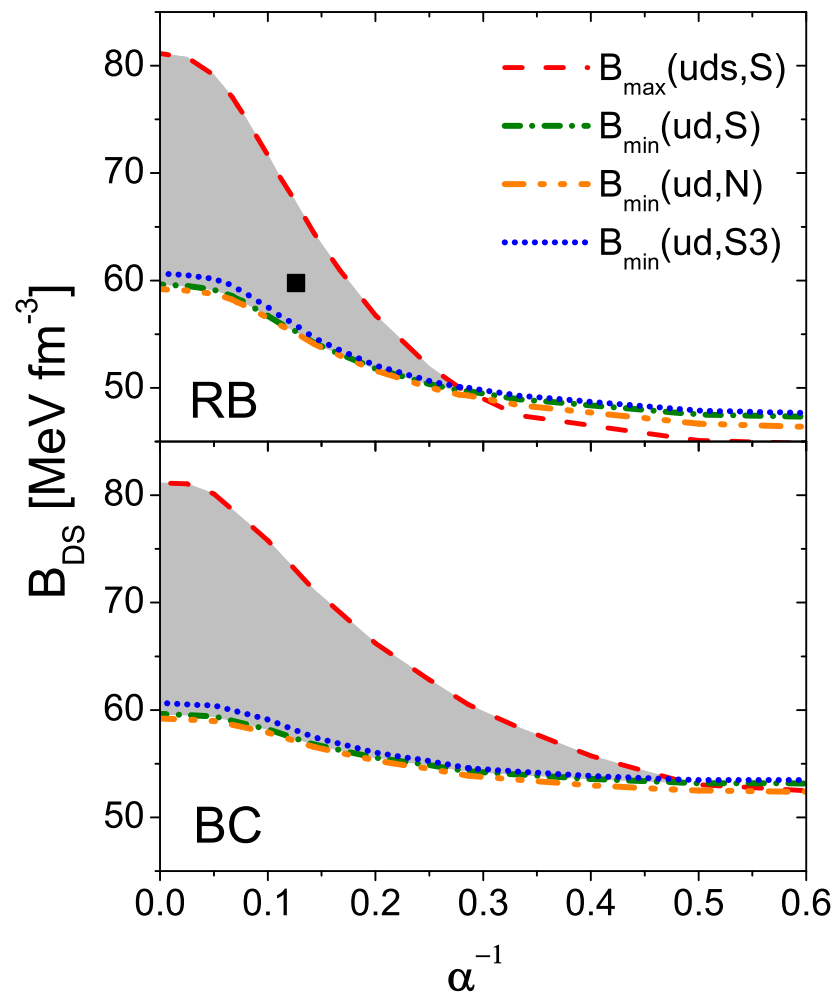

Fig. 5. (Color online) The allowed parameter space of $\alpha$ and $B_{\mathrm{DS}}$ for the SQM hypothesis in the DSM with RB (upper panel) or BC (lower panel) approximation, respecting the nuclear matter stability conditions. The various boundary curves, $B_{\min }$ indicating the lower limits due to the stability of ordinary nuclear matter, and $B_{\max }$ the upper limit for the stability of $\mathrm{SQM}$, are further discussed in the text. The marker refers to the choice of parameters for Fig. 6 .

With a large reduction rate of the interaction strength, $\alpha=10$ (lower panels), the dynamical mass of strange quarks decreases quickly and they can appear at small chemical potential. In such cases, if $B_{\mathrm{DS}}$ is too small (lower left panel), nuclear matter at $P=0$ would be unstable against $2 \mathrm{QM}$, which is inconsistent with physical reality. When $B_{\mathrm{DS}}$ is too large (lower right panel), at $P=0$ nuclear matter would be stable against $2 \mathrm{QM}$, but also against SQM. In all the above cases, the hypothesis of SQM would not be valid.

However, the parameters $\alpha$ and $B_{\mathrm{DS}}$ cannot be chosen arbitrarily, but are subject to severe constraints due to the fact that ordinary stable baryonic matter in our world is non-strange nuclear matter. We therefore have at least two quantitative constraints on the $2 \mathrm{QM}$,

$$
\begin{aligned}
\mu_{B}^{\mathrm{ud}, \mathrm{S}}(P=0) & >924 \mathrm{MeV}, \\
\mu_{B}^{\mathrm{ud}, \mathrm{N}}(P=0) & >939.4 \mathrm{MeV},
\end{aligned}
$$

obtained from the stability of symmetric nuclear matter (S) and neutral nuclear (neutron) matter (N) 9 . These two conditions enforce lower bounds on the parameter $B_{\mathrm{DS}}$ for each $\alpha$. On the other hand, for the hypothesis of SQM to be valid, SQM should be stable with respect 
to ordinary nuclear matter in an iron nucleus,

$$
\mu_{B}^{\mathrm{uds}, \mathrm{S}}(P=0)<930.4 \mathrm{MeV},
$$

which determines an upper bound on the parameter $B_{\mathrm{DS}}$ for each $\alpha$.

These constraints on the parameters $B_{\mathrm{DS}}$ and $\alpha$ are visualized in Fig. 5, where the shaded area contains the values $\left(\alpha, B_{\mathrm{DS}}\right)$ that produce SQM according to Eq. (13) (upper dashed red boundary curve), while respecting the stability conditions Eqs. (12 a,b) (lower dash-dotted green and dash-dot-dotted orange boundary curves). One can see that these two lower boundary lines are quite close to each other. In fact, even if we tighten the stability constraint and demand symmetric nuclear matter to remain in the nucleonic (BHF BOB) phase up to 3 times saturation density (dotted blue curve) the lower boundary does not change very much.

One notes that the figure establishes the absolute parameter bounds for possible SQM $\alpha>3.6,50<B_{\mathrm{DS}}<81$ (RB, top panel) and $\alpha>2.1,53<B_{\mathrm{DS}}<81$ (BC, bottom panel). In the MIT limit $\left(\alpha^{-1}=0\right)$ the bounds are $59<B_{\mathrm{DS}}<81$, and assuming massless strange quarks, one obtains $59<B_{\mathrm{DS}}<929$. From the plot one may conclude that an increasing interaction strength in the DSM effectively reduces the stability of QM, such that only small values of $B_{\mathrm{DS}}$ are permitted for increasing $\alpha^{-1}$. This is also consistent with the larger allowed parameter space with $\mathrm{BC}$ compared to RB approximation, since the former features a weaker interaction at finite chemical potential 64,65.

In particular, the DSM without in-medium dampening of the interaction $(\alpha=0)$ does not allow SQM, as it does neither provide the possibility of hybrid NSs 62,63 . This feature was also exposed in Ref. 62,63 by demonstrating that the density-dependent bag parameter

$$
B(\rho) \equiv \varepsilon(\rho)-\varepsilon_{\text {free }}(\rho)
$$

is a rapidly rising function of density, indicating the repulsive nature of the in-medium quark-quark interaction. This is in clear contrast with the MIT model, for example, where $B(\rho)$ is a constant by definition.

The lower boundaries in Fig. 5 represent universal stability conditions of ordinary nuclear matter that have to be respected by any quark model, whether modeling SQSs or ordinary (hybrid) quark NSs. In the latter case, there is a different upper limit $B_{\max }$, beyond which a transition to QM does not occur any more even for the heaviest NSs, i.e., hybrid NSs cannot be formed at all with the given quark model. This upper limit depends obviously on the chosen hadronic EOS and is much larger than the upper limit for the SQM phase, because the relevant phase transition might occur at very large pressure. In fact, in the case of the BOB EOS for the hadronic phase, this upper limit is beyond any reasonable range of the bag constant. For the same reason, the lower limit on the parameter $\alpha$ is much lower for ordinary hybrid stars than for SQSs, e.g., in Ref. 62, 63 an $\alpha_{\text {min }} \approx 0.5$ was considered.

Having determined the possible parameter values $\alpha$ and $B_{\mathrm{DS}}$, we illustrate a typical result in Fig. 6. which

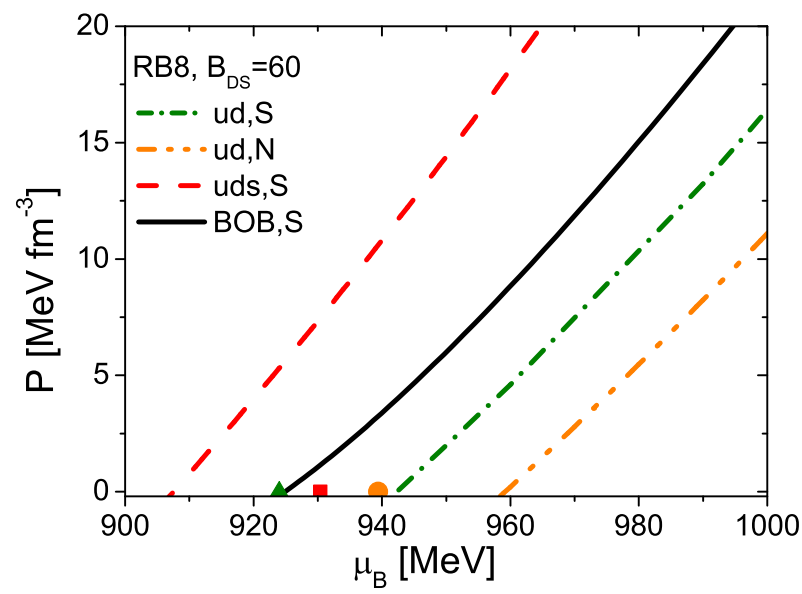

Fig. 6. (Color online) Pressure vs. baryon chemical potential of various EOSs. The stability conditions Eqs. (12) and (13) are symbolized by the markers.

shows the low-density EOS of symmetric nuclear matter (BOB,S), symmetric SQM (uds,S), and symmetric (ud,S) and neutral (ud,N) 2QM. The RB approximation and the parameter values $\alpha=8, B_{\mathrm{DS}}=60$ (see the black square marker in Fig. 5) are used.

\subsection{Structure of strange quark stars}

The relevant EOS for SQSs is the one of beta-stable and charge-neutral SQM, comprising a small electron fraction due to the finite strange quark mass, and we present that EOS in the form $P\left(\rho_{B}\right)$ in Fig. 7(top) for both the RB and $\mathrm{BC}$ models. In each case, we choose three sets of parameters $\left(\alpha, B_{\mathrm{DS}}\right)$, corresponding to the three extreme points of the allowed regions in Fig. 5. Note that two of these points (MIT limit) coincide for RB and BC model. For comparison, the nuclear BHF EOS is also shown. Different from nuclear matter, SQM approaches nonzero densities of about $0.25-0.4 \mathrm{fm}^{-3}$ at zero pressure. These are the surface densities of the corresponding SQSs, which decrease with decreasing $B_{\mathrm{DS}}$, i.e., increasing stability of QM. At higher densities, the pressure (and energy density) of SQM is much lower than that of nuclear matter.

With these EOSs we calculate the structure of bare SQSs. We treat a SQS as a spherically symmetric distribution of mass in hydrostatic equilibrium and obtain the stellar radius $R$ and the gravitational mass $M$ by the standard procedure of solving the TOV equations 81. We assume SQSs without crust, whereas for NSs we employ the BOB high-density BHF EOS joined with the ones by Negele and Vautherin 82 in the medium-density regime, and by Feynman-Metropolis-Teller [83] and BaymPethick-Sutherland 84 for the outer crust.

The results are shown in the lower panels of Fig. 7. The left (right) panel shows the star's gravitational mass dependent on the central baryon density (radius). Two of the quark model curves coincide with the MIT model, and 

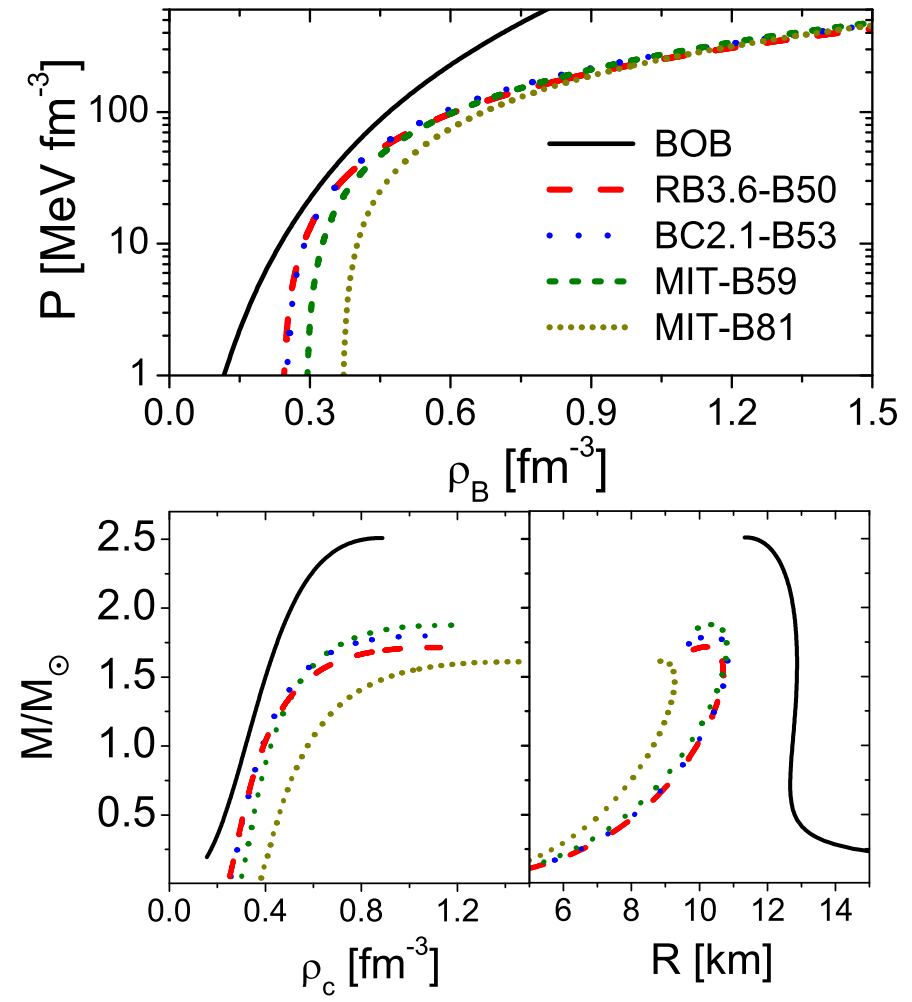

Fig. 7. (Color online) Upper plot: Pressure vs. baryon number density of SQS matter obtained with different EOSs. Lower plots: The corresponding gravitational mass - central density and mass - radius relations of SQSs $\left(M_{\odot}=2 \times 10^{33} \mathrm{~g}\right)$. See text for details.

in fact the configuration with minimum $B_{\mathrm{DS}}$ provides the largest maximum mass and radius of all EOSs. Increasing the bag constant or maintaining interacting quarks with finite $\alpha^{-1}$, reduces effectively the binding of QM and thus the values of the above physical quantities. In the lower right panel of Fig. 7 we find that typical radii of SQSs heavier than one solar mass are 10-11 km, a bit smaller than hadronic NS radii $11-13 \mathrm{~km}$, but close to a recent analysis on observed compact stars 85. Compared to other quark models [18,35, we obtain a much smaller range of possible radii and maximum masses of SQSs. This is due to the lower bound on the parameter $B_{\mathrm{DS}}$ from the constraint Eq. (12). For lower values of $B_{\mathrm{DS}}$ larger SQS masses can be reached, but the stability condition of ordinary nuclear matter is violated.

The single most relevant number characterizing a given NS model is probably the maximum mass, in view of the recent observation of heavy NSs 14,15,16. In order to address this issue, we show in Fig. 8 the maximum mass of SQSs for the allowed values of parameters $\left(\alpha, B_{\mathrm{DS}}\right)$, as determined in Fig. 5. In line with the repulsive character of the interaction in the DSM, the maximum mass increases with increasing binding of QM, i.e., with decreasing value of $B_{\mathrm{DS}}$ for fixed $\alpha$, or with increasing value of $\alpha$ for fixed $B_{\mathrm{DS}}$.

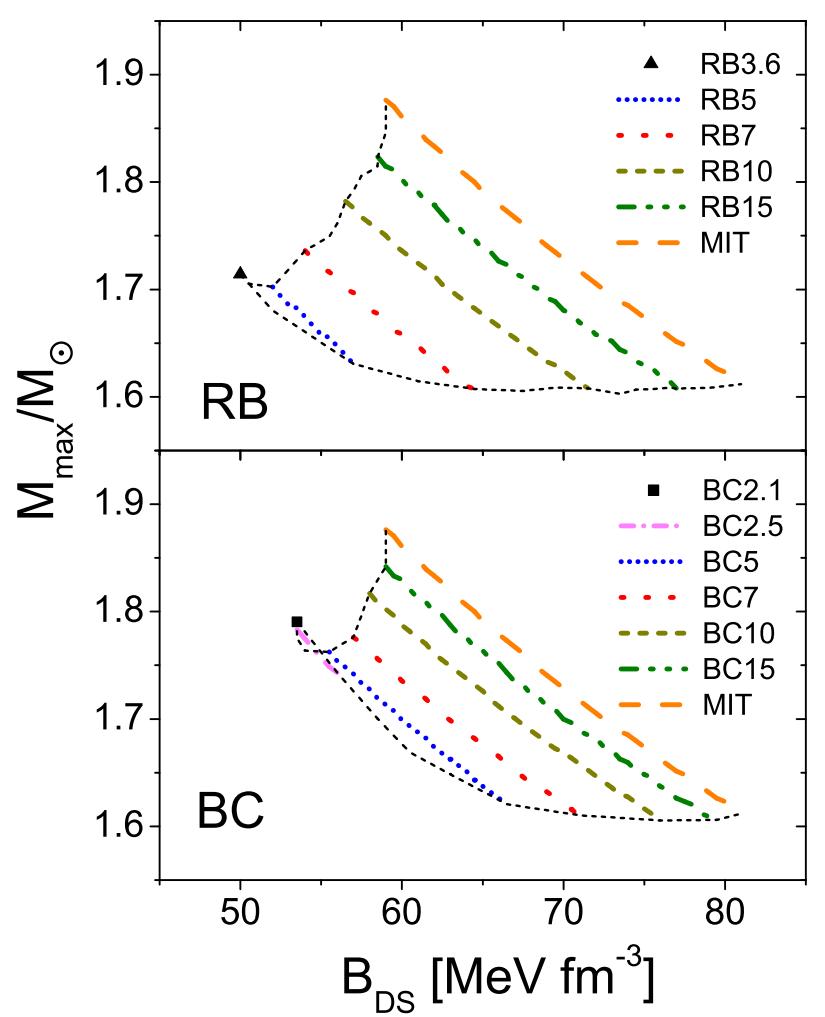

Fig. 8. (Color online) Maximum SQS mass $\left(M_{\odot}=2 \times 10^{33} \mathrm{~g}\right)$ in the DSM with RB (top panel) and BC (bottom panel) approximation, dependent on the value of $B_{\mathrm{DS}}$ for various fixed values of $\alpha$ within the constrained regions in Fig. 5

The globally largest value, $M_{\max } \approx 1.9 M_{\odot}$, is thus found in the MIT limit $\left(\alpha^{-1}=0\right)$ with the smallest possible bag constant $B_{\min }=59$. This value is smaller than the currently observed largest mass of compact stars 14 , [16]. Regarding finally the dependence on the remaining parameter $m_{s}$, it was shown in 3,26, that in the MIT limit a decreasing value of $m_{s}$ produces a larger permitted parameter space of the bag constant and an increase of $M_{\max }$; however, even for a vanishing strange quark mass $M_{\max }<1.95 M_{\odot}$.

In comparison with SQSs, the maximum mass of hybrid NSs can be much larger at larger $B_{\mathrm{DS}}$ and/or smaller $\alpha 62,63,64,65$. Due to the fact that a very stiff nuclear EOS (BOB) was used for their construction, in this case large maximum masses of about $2.5 M_{\odot}$ can be reached, which correspond to configurations where QM is only present in the very core of the star. However, this result cannot resolve the 'hyperon puzzle' [69,86, 87, which would impede the existence of heavy hadronic or hybrid NSs, where QM only appears at very large density. We leave detailed discussions of different realizations of hybrid NSs and their behavior in the whole parameter space to future work. 


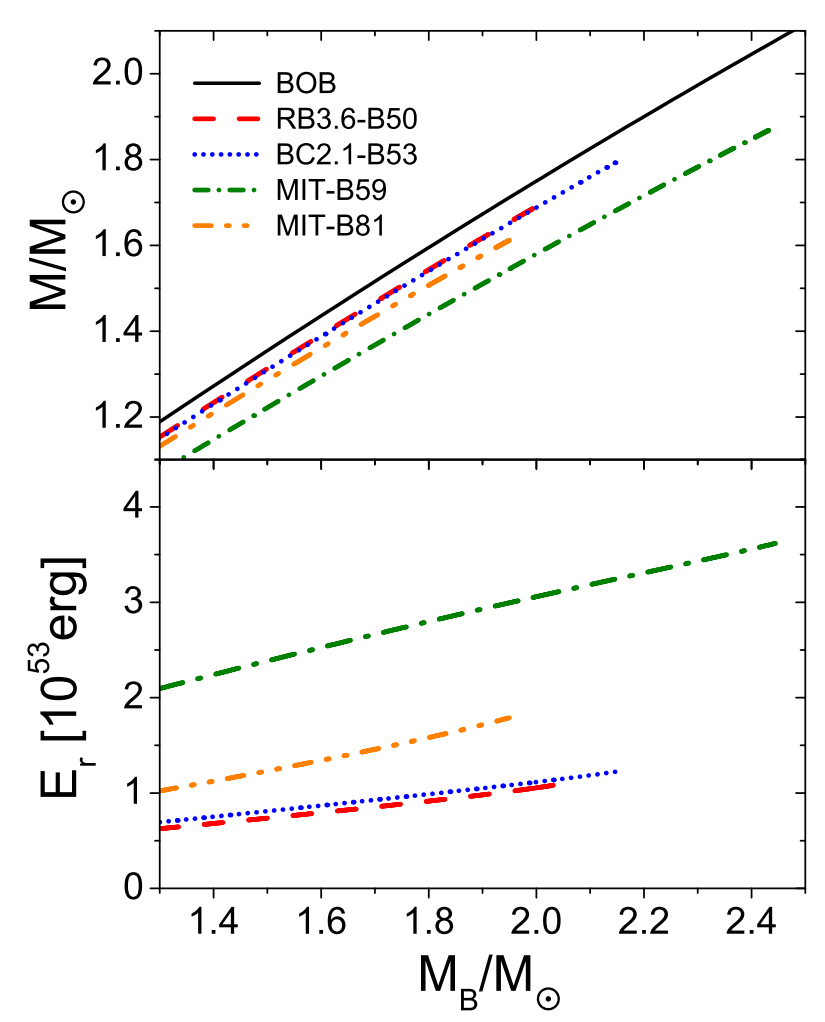

Fig. 9. (Color online) Upper panel: Gravitational mass vs. baryonic mass of compact stars for different EOSs. Lower panel: Energy release vs. baryonic mass in the conversion of NSs into SQSs with baryon number conservation.

\subsection{Conversion of neutron stars into strange quark stars}

As a last application, we consider the formation of a SQS from a pure metastable NS, which might occur once and if an initial seed of SQM has been formed in one of several hypothetical ways $[11,88,89,90$, for example in a two-step phase transition of nuclear matter to $2 \mathrm{QM}$ to SQM 91 92 , or in the combustion of hot nuclear matter [93, 94, 95 , 96, 97, 98.

This transition is accompanied by a huge energy release, which could be associated with the long gammaray bursts [99, 100,101] or the two-neutrino-burst scenario supernovas 102 . Here we simply assume that the total baryon number is conserved during the transition; then the energy release can be obtained from the mass difference between the pure NS and the SQS with the same total baryon number.

In Fig. 9] we show the relation between gravitational mass $M$ and baryonic mass $M_{B}$ of pure NSs and SQSs (upper panel) and the energy release $E_{r}$ in the conversion of NSs into SQSs with baryon number conservation (lower panel). The energy release depends on the DSM parameters and for a typical NS with $M=1.4 M_{\odot}$ can be $(0.8-2.5) \times 10^{53} \mathrm{erg}$, the maximum value of which is obtained for the MIT case $\left(\alpha^{-1}=0\right)$ with minimum value of the bag constant, i.e., for the strongest bound SQS, which also allows the largest maximum mass of SQSs, see Fig. 7.
These results are quantitatively similar to others in the literature, e.g., a range of $(1-4) \times 10^{53} \mathrm{erg}$ was obtained in Ref. [100]. As the NS mass increases, the energy release in the conversion also increases, up to $3.6 \times 10^{53} \mathrm{erg}$ for a NS with $M=2.1 M_{\odot}$ and the SQS obtained from the MITB59 case. For NSs with even larger masses, $M \approx(2.1-$ 2.5) $M_{\odot}$, there are no SQSs corresponding to the same baryon number. Therefore, it is impossible to convert such heavy NSs into SQSs with baryon number conservation, and they can only be converted into SQSs with a big loss of baryons, or into black holes.

\section{Conclusions}

We have investigated SQM and SQSs in our DSM for QM. For the hypothesis of SQM to be valid, i.e., SQM being stable against nuclear matter, while the latter is stable against 2QM, we obtained the allowed parameter space of $\alpha$ and $B_{\mathrm{DS}}$ in our DSM with RB or BC vertex. We found that SQM exists only for fairly low values of the bag parameter $B_{\mathrm{DS}}$ and furthermore a sufficiently strong damping of the in-medium effective interaction, expressed by a lower limit on the parameter $\alpha$. The strongest bound configurations correspond to the MIT bag-model limit, i.e., a vanishing in-medium effective interaction, with a minimal value of the bag constant.

This reinforces the idea that the full DSM does not provide a sufficiently attractive in-medium quark-quark interaction in order to create SQM. In fact that interaction turns out strongly repulsive in the DSM. This is in agreement with the NJL model, which does not allow SQS either, but in contrast to bag-model-type calculations (eventually including perturbative QCD corrections), which feature attractive interaction and strongly bound SQM with suitable parameter choices.

Outside the SQM parameter limits, hybrid two- or three-flavor NSs may exist in a much larger domain of the DSM parameter space, because in this case the upper limit on $B_{\mathrm{DS}}$ is established by a hadron-quark phase transition at high density in the NS core. Metastable twoflavor hybrid NSs may even coexist with SQSs. We will study these aspects in future work.

Then, in the allowed parameter space, we calculated the EOS of SQM and the corresponding structure of SQSs. We found that the maximum mass of SQSs is about $1.9 M_{\odot}$ and typical radii are $9-11 \mathrm{~km}$, while maximum masses of ordinary (hybrid) NSs are $2.5 M_{\odot}$ and $11-13 \mathrm{~km}$, depending on the hadronic EOS, though. We finally discussed the formation of SQSs due to the conversion from NSs and obtained energy releases as large as $3.6 \times 10^{53} \mathrm{erg}$.

For the future it will be important to establish direct estimates of the DSM model parameters in a more fundamental way from QCD, and in this way to clarify the qualitative differences between the different quark models mentioned above. 


\section{Acknowledgments}

We acknowledge useful comments from D. Blaschke and financial support from NSFC (11305144,11303023), Central Universities (CUGL 140609) in China. Partial support comes from "NewCompStar," COST Action MP1304.

\section{References}

1. A. R. Bodmer, Phys. Rev. D4, 1601 (1971).

2. E. Witten, Phys. Rev. D30, 272 (1984).

3. E. Farhi and R. L. Jaffe, Phys. Rev. D30, 2379 (1984).

4. D. D. Ivanenko and D. F. Kurdgelaidze, Astrophysics 1, 251 (1965).

5. N. Itoh, Prog. Theor. Phys. 44, 291 (1970).

6. H. Terazawa, INS-Report-336 (INS, University of Tokyo), May 1979.

7. N. K. Glendenning, Compact Stars, Nuclear Physics, Particle Physics, and General Relativity, 2nd ed. (Springer, New York, 2000).

8. F. Weber, Prog. Part. Nucl. Phys. 54, 193 (2005).

9. P. Haensel, A. Y. Potekhin, and D. G. Yakovlev, Neutron Stars 1 Equations of State and Structure (Springer, New York, 2007).

10. P. Haensel, J. L. Zdunik, and R. Schaeffer, Astron. Astrophys. 160, 121 (1986).

11. C. Alcock, E. Farhi, and A. V. Olinto, Astrophys. J. 310, 261 (1986).

12. I. Bombaci, Lecture Notes in Physics 578, 253 (2001).

13. F. Weber, G. A. Contrera, M. G. Orsaria, W. Spinella, and O. Zubairi, Mod. Phys. Lett. A29, 1430022 (2014).

14. P. B. Demorest, T. Pennucci, S. M. Ransom, M. S. E. Roberts, and J. W. T. Hessels, Nature 467, 1081 (2010).

15. E. Fonseca, T. T. Pennucci, J. A. Ellis, et al., arXiv:1603.00545

16. J. A. Antoniadis et al., Science 340, 6131 (2013).

17. V. Baluni, Phys. Rev. D17, 2092 (1978).

18. E. S. Fraga, R. D. Pisarski, and J. Schaffner-Bielich, Phys. Rev. D63, 121702(R) (2001).

19. A. Kurkela, P. Romatschke, and A. Vuorinen, Phys. Rev. D81, 105021 (2010).

20. E. S. Fraga, A. Kurkela, and A. Vuorinen, APJ Letters 781, L25 (2014).

21. J. F. Xu, G. X. Peng, F. Liu, De-Fu Hou, and Lie-Wen Chen, Phys. Rev. D92, 025025 (2015).

22. G. N. Fowler, S. Raha, and R. M. Weiner, Z. Phys. C9, 271 (1981).

23. S. Chakrabarty, Phys. Rev. D43, 627 (1991).

24. O. G. Benvenuto and G. Lugones, Phys. Rev. D51, 1989 (1995).

25. Ang Li, Ren-Xin Xu, and Ju-Fu Lu, MNRAS 402, 2715 (2010).

26. J. R. Torres and D. P. Menezes, Eur. Phys. Lett. 101, 42003 (2013).

27. M. Buballa, Nucl. Phys. A611, 393 (1996).

28. M. Buballa and M. Oertel, Phys. Lett. B457, 261 (1999).

29. M. Buballa, Phys. Rep. 407, 205 (2005).

30. K. Schertler, S. Leupold, and J. Schaffner-Bielich, Phys. Rev. C60, 025801 (1999).

31. T. Klähn, R. Lastowiecki, and D. Blaschke, Phys. Rev. D88, 085001 (2013).
32. T. Klähn and T. Fischer, APJ 810, 134 (2015).

33. A. Zacchi, R. Stiele, and J. Schaffner-Bielich, Phys. Rev. D92, 045022 (2015).

34. A. Peshier, B. Kämpfer, and G. Soff, Phys. Rev. C61, 045203 (2000).

35. Y.-L. Tian, Y. Yan, H. Li, X.-L. Luo, and H.-S. Zong, Phys. Rev. D85, 045009 (2012).

36. T. Zhao, Y. Yan, X.-L. Luo, and H.-S. Zong, Phys. Rev. D91, 034018 (2015).

37. C. D. Roberts and A. G. Williams, Prog. Part. Nucl. Phys. 33, 477 (1994).

38. R. Alkofer and L. von Smekal, Phys. Rep. 353, 281 (2001).

39. C. D. Roberts, M. S. Bhagwat, A. Höll, and S. V. Wright, Eur. Phys. J. Special Topics 140, 53 (2007).

40. C. S. Fischer and R. Williams, Phys. Rev. Lett. 103, 122001 (2009).

41. L. Chang and C. D. Roberts, Phys. Rev. Lett. 103, 081601 (2009).

42. L. Chang, I. C. Cloët, C. D. Roberts, S. M. Schmidt, and P. C. Tandy, Phys. Rev. Lett. 111, 141802 (2013).

43. G. Eichmann, I. C. Cloet, R. Alkofer, A. Krassnigg, and C. D. Roberts, Phys. Rev. C79, 012202 (2009).

44. G. Eichmann, R. Alkofer, A. Krassnigg, and D. Nicmorus, Phys. Rev. Lett. 104, 201601 (2010).

45. G. Eichmann, H. Sanchis-Alepuz, R. Williams, R. Alkofer, and C. S. Fischer, arXiv:1606.09602 [hep-ph].

46. C. D. Roberts and S. M. Schmidt, Prog. Part. Nucl. Phys. 45, S1 (2000).

47. A. Maas, Phys. Rep. 524, 203 (2013).

48. C. S. Fischer, Phys. Rev. Lett. 103, 052003 (2009);

49. C. S. Fischer and J. A. Mueller, Phys. Rev. D80, 074029 (2009).

50. C. S. Fischer, L. Fister, J. Luecker, and J. M. Pawlowski, Phys. Lett. B732, 273 (2014).

51. C. S. Fischer and J. Luecker, Phys. Lett. B718, 1036 (2013).

52. C. S. Fischer, J. Luecker, and C. A. Welzbacher, Phys. Rev. D90, 034022 (2014).

53. G. Eichmann, C. S. Fischer, and C. A. Welzbacher, Phys. Rev. D93, 034013 (2016).

54. S. X. Qin, L. Chang, H. Chen, Y. X. Liu, and C. D. Roberts, Phys. Rev. Lett. 106, 172301 (2011).

55. F. Gao, S.-X. Qin, Y.-X. Liu, C. D. Roberts, and S. M. Schmidt, Phys. Rev. D89, 076009 (2014).

56. F. Gao, J. Chen, Y.-X. Liu, S.-X. Qin, C. D. Roberts, and S. M. Schmidt, Phys. Rev. D93, 094019 (2016).

57. D. Nickel, J. Wambach, and R. Alkofer, Phys. Rev. D73, 114028 (2006).

58. D. Nickel, R. Alkofer, and J. Wambach, Phys. Rev. D74, 114015 (2006).

59. D. Müller, M. Buballa, and J. Wambach, Eur. Phys. J. A49, 96 (2013).

60. D. Müller, M. Buballa, and J. Wambach, arXiv:1603.02865 [hep-ph].

61. H. Chen, W. Yuan, L. Chang, Y. X. Liu, T. Klähn, and C. D. Roberts, Phys. Rev. D78, 116015 (2008).

62. H. Chen, M. Baldo, G. F. Burgio, and H.-J. Schulze, Phys. Rev. D84, 105023 (2011).

63. H. Chen, M. Baldo, G. F. Burgio, and H.-J. Schulze, D86, 045006 (2012).

64. H. Chen, J.-B. Wei, M. Baldo, G. F. Burgio, and H.-J. Schulze, Phys. Rev. D91, 105002 (2015). 
65. H. Chen, J.-B. Wei, M. Baldo, G. F. Burgio, and H.-J. Schulze, PoS CPOD2014 062 (2015).

66. T. Zhao, S.-S. Xu, Y. Yan, X.-L. Luo, X.-J. Liu, and H.-S. Zong, Phys. Rev. D92, 054012 (2015).

67. M. Baldo, I. Bombaci, and G. F. Burgio, Astron. Astrophys. 328, 274 (1997).

68. X. R. Zhou, G. F. Burgio, U. Lombardo, H.-J. Schulze, and W. Zuo, Phys. Rev. C69, 018801 (2004).

69. H.-J. Schulze, A. Polls, A. Ramos, and I. Vidana, Phys. Rev. C73, 058801 (2006).

70. Z. H. Li and H.-J. Schulze, Phys. Rev. C78, 028801 (2008).

71. J. S. Ball and T. W. Chiu, Phys. Rev. D22, 2542,2550 (1980); D23, 3085 (1981).

72. Y. Jiang, H. Chen, W.-M. Sun, and H.-S. Zong, JHEP 04, 014 (2013).

73. R. Alkofer, P. Watson, and H. Weigel, Phys. Rev. D65, 094026 (2002).

74. M. S. Bhagwat, M. A. Pichowsky, C. D. Roberts, and P. C. Tandy, Phys. Rev. C68, 015203 (2003).

75. C. D. Roberts, Prog. Part. Nucl. Phys. 61, 50 (2008).

76. L. Chang, Y.-X. Liu, M. S. Bhagwat, C. D. Roberts, and S. V. Wright, Phys. Rev. C75, 015201 (2007).

77. K.-L. Wang, S.-X. Qin, Y.-X. Liu, L. Chang, C. D. Roberts, and S. M. Schmidt, Phys. Rev. D86, 114001 (2012).

78. T. Klähn, C. D. Roberts, L. Chang, H. Chen, and Y. X. Liu, Phys. Rev. C82, 035801 (2010).

79. R. W. Haymaker, Riv. Nuovo Cim. 14, 1 (1991).

80. Y. Jiang, H. Gong, W.-M. Sun, and H.-S. Zong, Phys. Rev. D85, 034031 (2012).

81. S. L. Shapiro and S. A. Teukolsky, Black Holes, White Dwarfs, and Neutron Stars (John Wiley \& Sons, New York, 1983).

82. J. W. Negele and D. Vautherin, Nucl. Phys. A207, 298 (1973).

83. R. P. Feynman, N. Metropolis, and E. Teller, Phys. Rev. 75, 1561 (1949).

84. G. Baym, C. Pethick, and D. Sutherland, Astrophys. J. 170, 299 (1971).

85. F. Özel, D. Psaltis, T. Guver, G. Baym, C. Heinke, and S. Guillot, APJ 820, 28 (2016).

86. H.-J. Schulze and T. Rijken, Phys. Rev. C84, 035801 (2011).

87. Th. A. Rijken and H.-J. Schulze, Eur. Phys. J. A52, 21 (2016).

88. M. L. Olesen and J. Madsen, Phys. Rev. D49, 2698 (1994).

89. K. Iida and K. Sato, Phys. Rev. C58, 2538 (1998).

90. D. Logoteta, C. Providência, I. Vidaña, and I. Bombaci, Phys. Rev. C85, 055807 (2012).

91. Z. G. Dai, T. Lu, and Q. H. Peng, Acta Physica Sinica 42 , 8: 01210 (1993).

92. B. Niebergal, R. Ouyed, and P. Jaikumar, Phys. Rev. C82, 062801 (2010).

93. G. Lugones, O. G. Benvenuto, and H. Vucetich, Phys. Rev. D50, 6100 (1994).

94. G. Pagliara, M. Herzog, and F. K. Röpke, Phys. Rev. D87, 103007 (2013).

95. A. Drago and G. Pagliara, Phys. Rev. C92, 045801 (2015).

96. A. Ouyed, L. Welbanks, N. Koning, and R. Ouyed, arXiv:1504.03590.

97. S. Furusawa, T. Sanada, and S. Yamada, Phys. Rev. D 93, 043018 (2016).
98. S. Furusawa, T. Sanada, and S. Yamada, Phys. Rev. D 93, 043019 (2016).

99. K. S. Cheng and Z. G. Dai, Phys. Rev. Lett. 77, 1210 (1996).

100. I. Bombaci and B. Datta, Astrophys. J. 530, L69 (2000). 101. Z. Berezhiani, I. Bombaci, A. Drago, F. Frontera, and A. Lavagno, Astrophys. J. 586, 1250 (2003).

102. A. De Rujula, Phys. Lett. B193, 514 (1987). 